\title{
Fatal Recurrent Invasive Pulmonary Aspergillosis: A Rare Co-infection Case with Pulmonary Embolism in COPD
}

\author{
Shanshan Su, Ying Zhou, Yupeng Xie, Min Ye, Chengshui Chen and Yuping Li*
}

Department of Pulmonary Medicine, The First Affiliated Hospital of Wenzhou Medical University, Wenzhou, Zhejiang, China

\begin{abstract}
Background: Patients with severe chronic obstructive pulmonary disease (COPD) are becoming one of the main risk factors for invasive pulmonary aspergillosis (IPA). Furthermore, COPD patients are at a high risk for pulmonary embolism (PE). Both these two conditions, when COPD is concomitant with IPA or PE, carry a poor prognosis. The coexistence of these three fatal diseases is rare.

Case presentation: We report a case of recurrent IPA concomitant with PE in a 76-year-old male with GOLD 4 COPD, who presented as non-specific hypoxemia and dyspnea and did not respond to the antifungal therapy. This case shows that patients should be treated until resolution of all clinical and radiographic manifestations in case of reactivation and that 10 weeks of antifungal therapy may be inadequate. For the treatment failure of antifungals, we presume PE would aggravate ischemia and hypoxia in the fungal-infected tissue, thus limiting the access of antifungal drugs. It deserves further research to improve the efficacy of antifugals in IPA patients concurrent with PE.

Conclusion: A diagnosis of PE should be considered in COPD patients resistant to appropriate therapy such as bronchodilators and antibiotics. On the other hand, physicians should be alert for the relapse of IPA in COPD patients with prior IPA presented as fever and dyspnea not respond to appropriate therapy, and conduct diagnostic procedures promptly.
\end{abstract}

Keywords: Invasive pulmonary aspergillosis; Pulmonary embolism; Chronic obstructive pulmonary disease

\section{Introduction}

Worldwide there are 200,000 estimated annual cases of invasive aspergillosis [1]. At present, approximately $50 \%$ of all invasive aspergillosis are found in neutropenic hosts [2]. But patients with severe chronic obstructive pulmonary disease (COPD) are becoming one of the main risk groups for invasive pulmonary aspergillosis (IPA) [3]. Furthermore, COPD patients are at a high risk for pulmonary embolism (PE). Both these two conditions, when COPD is concomitant with IPA or PE, carry a very poor prognosis and are receiving more and more attention. Whereas, the coexistence of IPA and PE in one single COPD patient is rare. There is only one case about a COPD patient concomitant with $\mathrm{PE}$ and chronic necrotizing pulmonary aspergillosis (CNPA) in literature [4]. We report a case of recurrent IPA coexistent with PE, with a fatal outcome in an old male with COPD.

\section{Materials and Methods}

All specimens from airways, including sputum and bronchoalveolar lavage fluid, were cultured on conventional media and fungal media. Aspergillus isolates were identified using standard morphological procedures. The galactomannan level was determined in serum samples. We used a cut-off of $\geq 0.5 \mathrm{ng} / \mathrm{mL}$ to define positivity.

The patient was examined on a helical CT system (Siemens Medical Solutions, Germany) with a slice thickness of $5 \mathrm{~mm}$. And for computed tomography pulmonary angiography contrast agent was Iopamidol, with a speed of $4 \mathrm{ml} / \mathrm{s}$.

\section{Case Report}

In January 2015, a 76-year-old ex-smoker was referred to our hospital with a 2-months history of increase in cough, purulent expectoration, exertional dyspnea and pyrexia. He had COPD history with regular use of long-acting inhaled bronchodilators (Salmeterol/Fluticasone 50 $\mu \mathrm{g} / 500 \mu \mathrm{g}$ and Tiotropium Bromide). And when symptoms exacerbated occasionally, a short course of systemic glucocorticoid was prescribed orally or intravenously. He once admitted to our hospital for an acute exacerbation in 2009 when the spirometry revealed a GOLD 4 COPD, with a very severe airflow obstruction (forced expiratory volume in one second $\left(\mathrm{FEV}_{1}\right)$ : $0.60 \mathrm{~L}, 28.0 \%$ of predicted; forced vital capacity (FVC): 1.27 L, $45.2 \%$ of predicted; $\mathrm{FEV}_{1} / \mathrm{FVC}$ : $47.2 \%$ ). Additionally, in September 2012, he had once been diagnosed as probable IPA at a local hospital according to the Bulpa criteria [5], since the chest CT scan showed a cavity in the right upper lobe (Figure 1) and the sputum culture was positive for Aspergillus fumigatus for more than one time. Therefore, the patient underwent antifungal therapy (intravenous itraconazole for two weeks, followed by oral itraconazole for two months). As symptoms improved, he withdrew the drugs with no doctors' order and follow-up. Comorbidities included hypertension and diabetes mellitus with no drug use. In December 2014, when the patient was admitted at the local hospital, a chest CT scan showed a fibrotic lesion in right upper lobe, considered as a scar healed from the previous infected cavity (Figure 2), with emphysematous change. Subsequently, a diagnosis of 'acute exacerbation of COPD' was made and antibiotics (imipenem) and methylprednisolone were administered, which gradually improve the symptoms in 2 weeks. However, the condition deteriorated with similar symptoms 4 days later, while readministration of antibiotics and corticosteroids turned out ineffective. Therefore, the patient was referred to our hospital.

On admission, he was conscious but cyanosed, with moderate nutrition status, body temperature $38^{\circ} \mathrm{C}$, blood pressure $107 / 74 \mathrm{mmHg}$,

*Corresponding author: Yu-Ping Li, MD, Department of Pulmonary Medicine The First Affiliated Hospital of Wenzhou Medical University, Wenzhou, Zhejiang 325015, China, Tel: (86) 0577-5557-9273; Fax: (86) 0577-5557-9273; E-mail: wzliyp@163.com

Received February 20, 2016; Accepted April 14, 2016; Published April 18, 2016

Citation: Su S, Zhou Y, Xie Y, Ye M, Chen C, et al. (2016) Fatal Recurrent Invasive Pulmonary Aspergillosis: A Rare Co-infection Case with Pulmonary Embolism in COPD. J Pulm Respir Med 6: 333. doi:10.4172/2161-105X.1000333

Copyright: (c 2016 Su S, et al. This is an open-access article distributed under the terms of the Creative Commons Attribution License, which permits unrestricted use, distribution, and reproduction in any medium, provided the original author and source are credited. 
Citation: Su S, Zhou Y, Xie Y, Ye M, Chen C, et al. (2016) Fatal Recurrent Invasive Pulmonary Aspergillosis: A Rare Co-infection Case with Pulmonary Embolism in COPD. J Pulm Respir Med 6: 333. doi:10.4172/2161-105X.1000333

Page 2 of 4

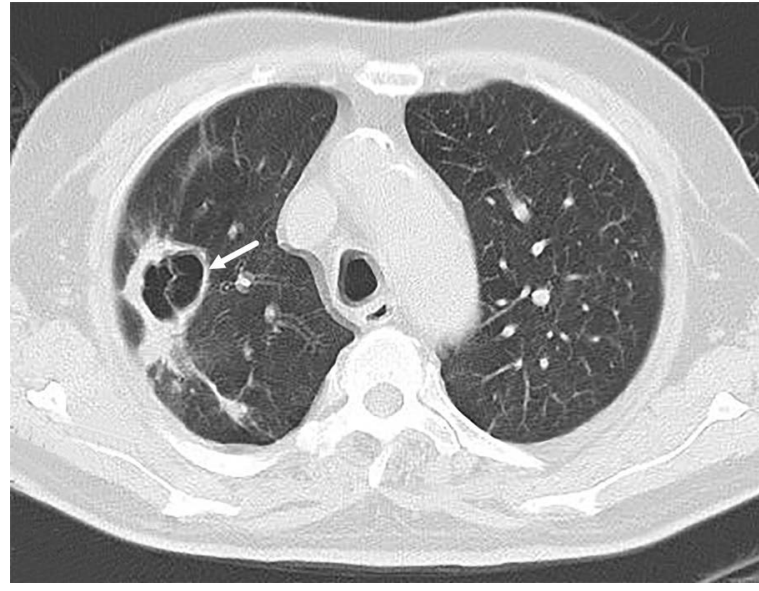

Figure 1: Chest CT scan (2012-9-28) revealed a cavity in right uppe lobe (white arrow, size $2.8 \times 2.1 \mathrm{~cm}$ ), which led to the diagnosis of probable IPA and COPD, combined with the positive finding in sputum culture for $A$. fumigatus.

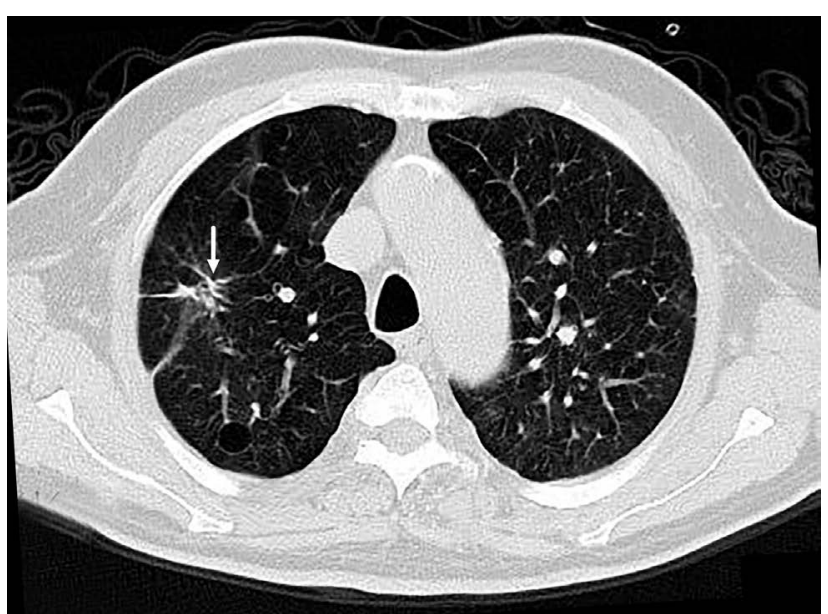

Figure 2: Chest CT scan (2014-12-6) showed a fibrotic lesion in right upper lobe (white arrow).

respiratory rate 22 breaths/min, heart rate 116 beats/min and oxygen saturation $90 \%$ on room air. Physical examination revealed barrel chest, with obvious use of accessory respiratory muscle. Both lower lung crackles and moderate bilateral lower limb edema were found.

Laboratory tests showed neutrophilia $(16650 / \mu \mathrm{l}$, normal range 1800-6300/ $/ \mathrm{l})$, lymphopenia $(400 / \mu \mathrm{l}$, normal range $1100-3200 / \mu \mathrm{l})$, erythrocytosis $\left(6.22 \times 10^{3} / \mu \mathrm{l}\right.$, normal range $\left.4.3 \times 10^{3}-5.8 \times 10^{3} / \mu \mathrm{l}\right)$, elevated serum C-reactive protein $(172 \mathrm{mg} / \mathrm{L}$, normal range $0-8 \mathrm{mg} / \mathrm{L})$, procalcitonin levels $(1.03 \mathrm{ng} / \mathrm{mL}$, normal range $0-0.5 \mathrm{ng} / \mathrm{mL})$, D-dimer assay $(1.41 \mathrm{mg} / \mathrm{L}$, normal range $0-0.5 \mathrm{mg} / \mathrm{L})$ and brain natriuretic peptide $(2305 \mathrm{pg} / \mathrm{mL}$, normal range $0-100 \mathrm{pg} / \mathrm{mL})$. Arterial blood gas analysis revealed $\mathrm{pH} 7.36, \mathrm{PO}_{2} 58.9 \mathrm{mmHg}$ and $\mathrm{PCO}_{2} 76.5 \mathrm{mmHg}\left(\mathrm{FiO}_{2}\right.$ 0.33). Biochemical tests revealed elevated fasting blood glucose (9.1 $\mathrm{mmol} / \mathrm{L}$, normal range 3.9-6.1 $\mathrm{mmol} / \mathrm{L}$ ) with normal liver and renal function tests. The serum tumor markers and troponin were within normal range, while sputum smear for acid-fast bacillus and T-SPOT. TB were negative. Two-dimensional echocardiography, done at local hospital, had revealed right ventricular and atrial dilatation, tricuspid regurgitation and an estimated pulmonary artery systolic pressure of $77 \mathrm{mmHg}$. Notably, lower limb venous duplex ultrasonography had revealed vein thrombosis in the superficial femoral vein.

As the broad-spectrum antibiotics with nebulized bronchodilators did not improve the symptoms and venous ultrosonography had a positive result, a pulmonary embolism was suspected and CT pulmonary angiogram (CTPA) was conducted which revealed filling defects in the right main pulmonary artery extending into the upper and proximal part of lower lobar branches (Figure 3). Unexpectedly, there existed a cavity with consolidation around in right upper lobe, which was just at the same site of previous cavity and supplied by the occluded vessel (Figures 3 and 4). It seemed reasonably to suspect reactivation of fungal infection, so serum galactomannan (GM) and $\beta-(1,3)-D$ glucan assays were tested. Meanwhile, an anticoagulant therapy with subcutaneous low-molecular-weight heparin (Fraxiparine, $0.1 \mathrm{ml} / 10 \mathrm{~kg}$ every 12 hours) were given immediately, followed by Warfarin (with doses adjusted to maintain the INR at a target of 2.5, range 2.0-3.0). Four days later GM assay turned out 0.58 (cut-off 0.5 ), with normal $\beta$-(1,3)-D-glucan assay. Besides, the sputum culture (sample collected at admission) was positive for A. fumigatus twice and negative for bacteria.

According to the fever, cavity in the CT scan, positive serum GM and positive results for A. fumigatus in sputum, the diagnosis of recurrent probable IPA was confirmed referring to the Bulpa criteria [5]. Immediately an antifungal therapy with intravenous voriconazole $(6 \mathrm{mg} / \mathrm{kg}$ every 12 hours in the first 24 hours, followed by $4 \mathrm{mg} / \mathrm{kg}$ every 12 hours) was given in addition to anticoagulant therapy. However, seven days later after initiation of antifungal treatment, the condition of the patient deteriorated with a hypoxemia, hypercapnia, consciousness, high temperature rising to $42^{\circ} \mathrm{C}$ and septic shock. The patient received intubation, mechanical ventilation and bedside bronchoscopy examination. The culture of bronchoalveolar lavage fluid (BALF) and brushing in right upper lobe all revealed positive for A. fumigatus. Although aggressive treatment was applied, the patient died in two days.

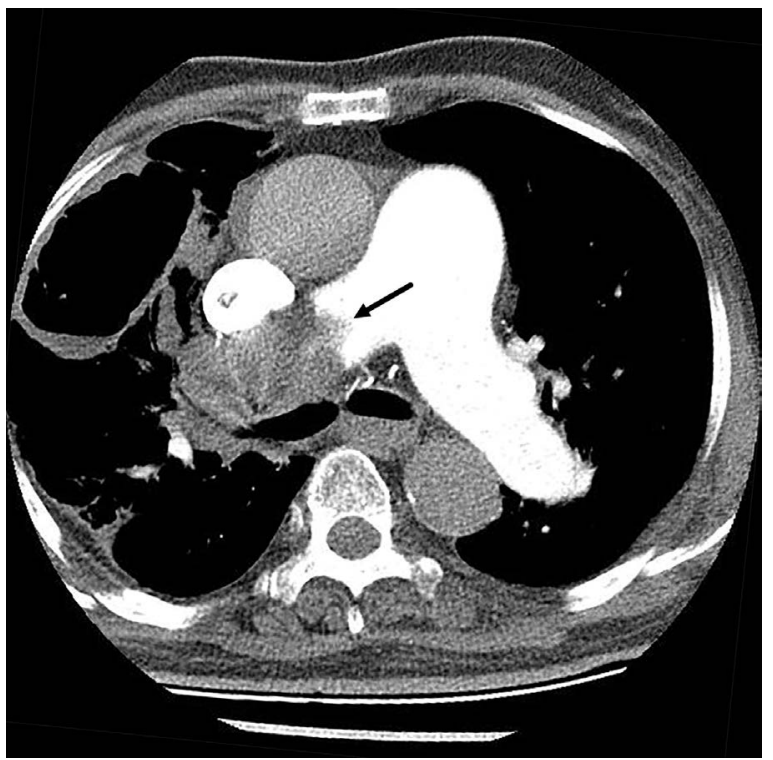

Figure 3: CTPA (2015-1-9) showed filling defects in the right main pulmonary artery (black arrow) extending into the upper and proximal part of lower lobar branches. 


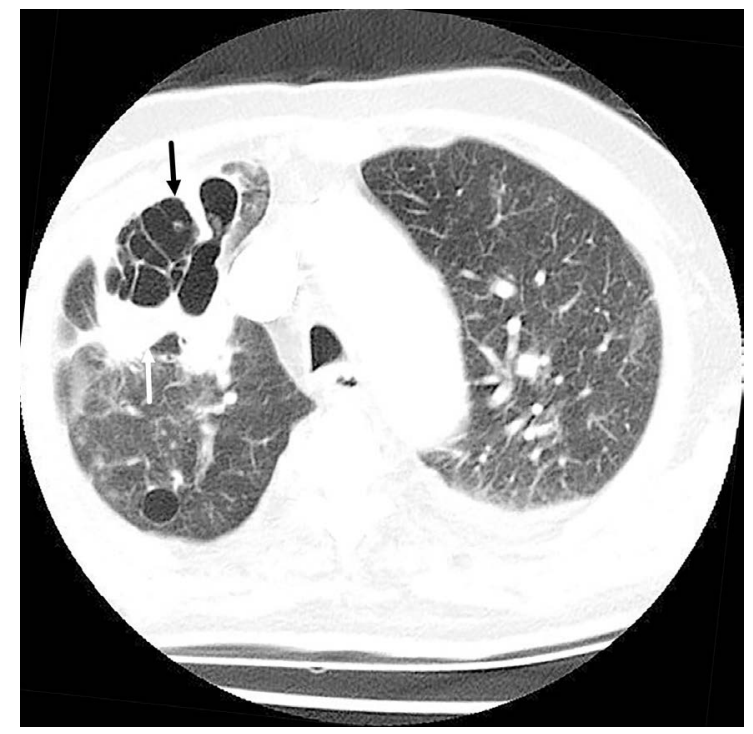

Figure 4: Chest CT scan (2015-1-9) revealed a cavity (black arrow) with consolidation around (white arrow) in right upper lobe, which was at the same site of previous cavity showed by Figure 1 . And the largest crosssectional size of cavity was $6.6 \times 3.5 \mathrm{~cm}$.

\section{Discussion}

This report describes the difficult circumstances where recurrent IPA, PE and COPD coexist in a single patient in the clinical practice.

The COPD patient presented fever and dyspnea, which are common symptoms in acute exacerbation and could be improved by appropriate therapy. In view of the high prevalence of PE in COPD patients with non-specific clinical signs and the mortality risk associated with undiagnosed PE [6], clinicians should consider carefully for the diagnosis of concomitant PE in COPD patients resistant to appropriate therapy. On the other hand, in severe COPD patients with prior IPA history, the presence of antibiotic resistant pyrexia and breathlessness is suggestive of recurrent IPA, especially those improve initially but aggravate soon. As the chest imaging may be normal in the early stage, a repeat CT scan shall be conducted timely to visualize alterations of the lung parenchyma to confirm the suspicion of IPA, as well as other diagnostic procedures such as sputum culture and serum GM test.

Duration of antifungal therapy for IPA is not optimally defined. The guideline [7] recommends that treatment be continued for a minimum of 6-12 weeks based on the data in neutropenic hosts. And some experts [8] suggest that duration of treatment in non-neutropenic hosts be a minimum of 12 weeks, depending on the degree of ongoing immunosuppression and resolution of lesions revealed by repeat CT scan. However, the data specific for COPD patients are lacking. The patient, when first diagnosed as probable IPA in September 2012, had received about 10 weeks of antifungals and withdrew the drugs without a repeat CT scan or follow-up. Whether the lesion had resolved when drugs were withdrawn was unclear, though the chest CT done in 2014 showed that cavity had resolved leaving a fibrotic lesion. This may imply that IPA in COPD patients should be treated adequately until resolution or stabilization of all clinical and radiographic manifestations in case of reactivation of IPA, especially in acute exacerbations of COPD receiving systemic steroids and broad-spectrum antibiotics.

The patient did not respond to antifungal therapy of standard doses of voriconazole, with a measured trough concentration of 4.68 $\mu \mathrm{g} / \mathrm{ml}(1-5 \mu \mathrm{g} / \mathrm{ml}$ have been proposed as optimal) [9]. This refractory condition might be explained by concomitance of PE. Severe COPD patients were complicated with pulmonary hypertension and right ventricular dilation, resulting from remodeling and destruction of pulmonary vascular bed, which indicates decreased pulmonary blood flow and poor hemodynamic reserve. When concurrent with $\mathrm{PE}$, apart from the physical obstruction to flow, PE triggers the release of vasoactive substances, resulting in vasoconstriction and vasospasm, which aggravate ischemia and hypoxia in the fungal-infected tissue, thus limiting the trafficking of antifungals. Besides, IPA is characterized by angioinvasion, limiting the access of antifungal drugs to the site of infection and impaired fungal clearance [10]. Researchers have hypothesized that $A$. fumigatus metabolites inhibit compensatory angiogenesis of hosts leading to worsening tissue hypoxia, sequestration of infected tissue, and limitation of antifungals access [11]. Furthermore, one study [12] has confirmed that the repletion of proangiogenic growth factors (VEGF and bFGF) would reverse angiogenesis inhibition at the site of infection, potentiate the in vivo activity of antifungals (amphotericin B), and improve survival rates in a neutropenic mouse model of IPA. However, the result in the neutropenic mouse model of IPA may be inconsistent with that in non-neutropenic hosts, such as COPD, which needs further research.

\section{Conclusion}

We present a devastating case with relapsing IPA and PE coexisting in COPD. This report reminds clinicians that a diagnosis of PE should be considered in COPD patients resistant to appropriate therapy. Meanwhile, in COPD patients with prior IPA presented as antibiotic resistant symptoms, physicians should be alert for the relapse of IPA and conduct diagnostic procedures promptly. And it deserves further research to improve the efficacy of antifungal drugs in IPA patients when concurrent with PE and COPD.

\section{Conflicts of Interest}

The authors have stated explicitly that there are no conflicts of interest in connection with this article.

\section{Acknowledgement}

This work is supported by a Project Grant from the Science \& Technology Bureau of Zhejiang Province (2013C33172).

\section{References}

1. Brown GD, Denning DW, Gow NA, Levitz SM, Netea MG, et al. (2012) Hidden killers: human fungal infections. Sci Transl Med 4: 165rv13.

2. Schmiedel Y, Zimmerli S (2016) Common invasive fungal diseases: an overview of invasive candidiasis, aspergillosis, cryptococcosis, and Pneumocystis pneumonia. Swiss Med Wkly 146: w14281.

3. Guinea J, Torres-Narbona M, Gijon P, Munoz P, Pozo F, et al. (2010) Pulmonary aspergillosis in patients with chronic obstructive pulmonary disease: incidence risk factors, and outcome. Clin Microbiol Infect 16: 870-877.

4. Terzano C, Conti V, Petroianni A, Paone G (2013) An "alternative" clinical course of COPD exacerbation and pulmonary embolism. Eur Rev Med Pharmacol Sci 17: $3341-3346$.

5. Bulpa P, Dive A, Sibille $Y$ (2007) Invasive pulmonary aspergillosis in patients with chronic obstructive pulmonary disease. Eur Respir J 30: 782-800.

6. Rizkallah J, Man SF, Sin DD (2009) Prevalence of pulmonary embolism in acute exacerbations of COPD: a systematic review and metaanalysis. Chest 135: 786-793.

7. Walsh TJ, Anaissie EJ, Denning DW, Herbrecht R, Kontoyiannis DP, et al (2008) Treatment of aspergillosis: clinical practice guidelines of the Infectious Diseases Society of America. Clin Infect Dis 46: 327-360. 
Citation: Su S, Zhou Y, Xie Y, Ye M, Chen C, et al. (2016) Fatal Recurrent Invasive Pulmonary Aspergillosis: A Rare Co-infection Case with Pulmonary Embolism in COPD. J Pulm Respir Med 6: 333. doi:10.4172/2161-105X.1000333

8. Kosmidis C, Denning DW (2015) The clinical spectrum of pulmonary aspergillosis. Thorax 70: 270-277

9. Elewa H, El-Mekaty E, El-Bardissy A, Ensom MH, Wilby KJ (2015) Therapeutic Drug Monitoring of Voriconazole in the Management of Invasive Fungal Infections: A Critical Review. Clin Pharmacokinet 54: 1223-1235.

10. Paterson PJ, Seaton S, Prentice HG, Kibbler CC (2003) Treatment failure in invasive aspergillosis: susceptibility of deep tissue isolates following treatment with amphotericin B. J Antimicrob Chemother 52: 873-876.
11. Ben-Ami R (2013) Angiogenesis at the mold-host interface: a potential key to understanding and treating invasive aspergillosis. Future Microbiol 8: 14531462.

12. Ben-Ami R, Albert ND, Lewis RE, Kontoyiannis DP (2013) Proangiogenic growth factors potentiate in situ angiogenesis and enhance antifungal drug activity in murine invasive aspergillosis. J Infect Dis 207: 1066-1074. 